\title{
ON MAXIMUM PRINCIPLES FOR DIFFUSION IN THE PRESENCE OF THREE DIFFUSION PATHS
}

\author{
ALEXANDER I. LEE and JAMES M. HILL'
}

(Received 11 December 1981; revised 3 June 1982)

\begin{abstract}
This note examines maximum principles for systems of parabolic partial differential equations describing diffusion in the presence of three diffusion paths. The particular system under consideration arises from a random walk model. For a more general system constraints on the various constants are given which guarantee maximum principles. Remarkably, the physical system arising from the random walk model automatically satisfies these constraints.
\end{abstract}

\section{Introduction}

The diffusion of ions and point defects in metals which are comprised of a continuous distribution of high-diffusivity paths such as grain boundaries and dislocations has recently been modelled by Aifantis [1,2]. In the general theory it is assumed that each point of the medium is simultaneously occupied by $n$ diffusion paths and the concentrations in each diffusion path are governed by a system of $n$ parabolic partial differential equations. Recently, Hill [8] presented a simple discrete random walk model for diffusion which, in the continuous limit, gives rise to this system of partial differential equations. Extensive study has been completed for diffusion in the presence of two diffusion paths and the reader is referred to Aifantis and Hill [3], Hill and Aifantis [10] and Hill [9]. Further applications for this system of parabolic differential equations, when $n=2$, arise in the theories of seepage of homogeneous liquids in fissured rocks (Barenblatt, Zheltov and Kochina [4]), the conduction of heat in heterogeneous media (Rubinstein [13]), and the transport of water through plant tissue (Molz [12]). In this

\footnotetext{
'Department of Mathematics, Unversity of Wollongong, Wollongong, N.S.W. 2500.

(C) Copyright Australian Mathematical Society 1983
} 
note maximum principles are examined for the system of equations which describes diffusion in the presence of three diffusion paths, namely

$$
\left.\begin{array}{l}
\partial u_{1} / \partial t=D_{1} \nabla^{2} u_{1}-\left(a_{21}+a_{31}\right) u_{1}+a_{12} u_{2}+a_{13} u_{3}, \\
\partial u_{2} / \partial t=D_{2} \nabla^{2} u_{2}+a_{21} u_{1}-\left(a_{12}+a_{32}\right) u_{2}+a_{23} u_{3} \\
\partial u_{3} / \partial t=D_{3} \nabla^{2} u_{3}+a_{31} u_{1}+a_{32} u_{2}-\left(a_{13}+a_{23}\right) u_{3}
\end{array}\right\}
$$

for $u_{1}(x, t), u_{2}(x, t)$ and $u_{3}(x, t)$ which are concentíatioñ and täkén iu be non-negative. The diffusivities $D_{1}, D_{2}$ and $D_{3}$ are non-negative constants. The constants $a_{i j}(i, j=1,2,3 ; i \neq j)$ represent transition probabilities in the random walk model of Hill [8] and are therefore non-negative.

In this note we develop maximum principles for the more general non-conservative system given by

$$
\left.\begin{array}{l}
\partial u_{1} / \partial t=D_{1} \nabla^{2} u_{1}-a_{11} u_{1}+a_{12} u_{2}+a_{13} u_{3}, \\
\partial u_{2} / \partial t=D_{2} \nabla^{2} u_{2}+a_{21} u_{1}-a_{22} u_{2}+a_{23} u_{3}, \\
\partial u_{3} / \partial t=D_{3} \nabla^{2} u_{3}+a_{31} u_{1}+a_{32} u_{2}-a_{33} u_{3},
\end{array}\right\}
$$

where $a_{11}, a_{22}$ and $a_{33}$ are further non-negative constants. We show that sufficient conditions on the constants which guarantee maximum principles for the system (1.2) are contained in

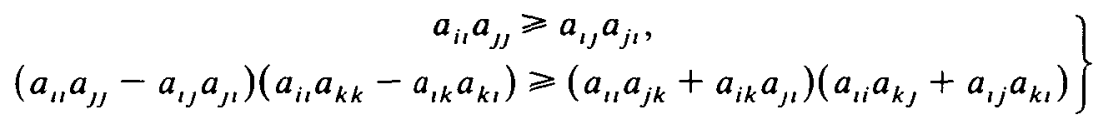

for $i, j, k=1,2,3 ; i \neq j, j \neq k, k \neq i$. For the system (1.1) we find that these inequalities are trivially satisfied upon substituting in (1.3)

$$
a_{u}=\sum_{\substack{j=1 \\ j \neq 1}}^{3} a_{j u} \quad(i=1,2,3)
$$

Thus, remarkably, a maximum principle is available for the physical system (1.1) without imposing any further conditions on the constants $a_{i j}$. These results are given as theorems in the following section.

\section{Maximum principles}

In this section maximum principles are obtained for the systems (1.1) and (1.2). The concentrations $u_{1}, u_{2}$ and $u_{3}$ are defined over a bounded domain $\Omega$ in the $\mathbf{x}$-space and for the finite time interval, $0 \leqslant t \leqslant T$. This region of $\mathbf{x}-t$ space is 
denoted by

$$
\mathbf{R}=\{(\mathbf{x}, t): \mathbf{x} \in \Omega \cup \partial \Omega, 0 \leqslant t \leqslant T\},
$$

where $\partial \Omega$ is the piecewise continuously differentiable surface of $\Omega$. Theorem 2.1 states that for particular conditions on the concentrations, at least one of the concentrations in the system (1.2) must attain its maximum on the boundary of $\mathbf{R}$, whenever conditions (1.3) hold. Theorem 2.2 is the corresponding result for the system (1.1). The following lemma and definition are required.

LEMMA. Let $A, B$ and $b_{i j}(i, j=1,2)$ be positive constants. If

$$
-b_{11} A+b_{12} B>0 \text { and } b_{21} A-b_{22} B>0,
$$

then

$$
b_{12} b_{21}-b_{11} b_{22}>0
$$

Proof. The first of (2.2) implies $B>b_{11} b_{12}^{-1} A$. The second of (2.2) implies $A>b_{22} b_{21}^{-1} B$. The elimination of either $A$ or $B$ gives $b_{11} b_{22} b_{12}^{-1} b_{21}^{-1}<1$. This implies (2.3).

DEFINITION. We say that $\left\{u_{1}, u_{2}, u_{3}\right\} \in H$ in $S$ if and only if

(i) $\left\{u_{1}, u_{2}, u_{3}\right\} \in C^{2}$ in $S$, and

(ii) $\left\{u_{1}, u_{2}, u_{3}\right\}$ satisfy (1.2) in $S$, where $S$ is an arbitrary region of the $\mathbf{x}-t$ space, and $C^{2}$ denotes the set of functions which are continuous together with their derivatives up to second order.

THEOREM 2.1. At least one of $u_{1}, u_{2}$ and $u_{3}$ attains its maximum on the boundary of $\mathbf{R}$ if the following hold:

(i) $\left\{u_{1}(\mathbf{x}, t), u_{2}(\mathbf{x}, t), u_{3}(\mathbf{x}, t)\right\} \in H$ if $\mathbf{x} \in \Omega, 0<t<T$,

(ii) $\left\{u_{1}(\mathbf{x}, t), u_{2}(\mathbf{x}, t), u_{3}(\mathbf{x}, t)\right\}$ are continuous if $(\mathbf{x}, t) \in \mathbf{R}$,

(iii) $\left\{u_{1}(\mathbf{x}, t), u_{2}(\mathbf{x}, t), u_{3}(\mathbf{x}, t)\right\} \geqslant 0$ if $(\mathbf{x}, t) \in \mathbf{R}$,

(iv) $a_{11} a_{22}>a_{12} a_{21}, a_{11} a_{33}>a_{13} a_{31}$,

(v) $\left(a_{11} a_{22}-a_{12} a_{21}\right)\left(a_{11} a_{33}-a_{13} a_{31}\right) \geqslant\left(a_{11} a_{23}+a_{13} a_{21}\right)\left(a_{11} a_{32}+a_{12} a_{31}\right)$.

Proof. The substitution of the new concentrations

$$
\left.\begin{array}{l}
u_{1}^{*}=u_{1}+\varepsilon e^{x\left(a_{11} / D_{1}\right)^{1 / 2}}, \\
u_{2}^{*}=u_{2}+\varepsilon e^{x\left(a_{22} / D_{2}\right)^{1 / 2}}, \\
u_{3}^{*}=u_{3}+\varepsilon e^{x\left(a_{33} / D_{3}\right)^{1 / 2}}
\end{array}\right\}
$$


for $\varepsilon>0$, into (1.2) yields

$$
\left.\begin{array}{c}
D_{1} \nabla^{2} u_{1}^{*}-\partial u_{1}^{*} / \partial t-a_{11} u_{1}^{*}+a_{12} u_{2}^{*}+a_{13} u_{3}^{*} \\
=\varepsilon\left[a_{12} e^{x\left(a_{22} / D_{2}\right)^{1 / 2}}+a_{13} e^{x\left(a_{33} / D_{3}\right)^{1 / 2}}\right]>0, \\
D_{2} \nabla^{2} u_{2}^{*}-\partial u_{2}^{*} / \partial t+a_{21} u_{1}^{*}-a_{22} u_{2}^{*}+a_{23} u_{3}^{*} \\
=\varepsilon\left[a_{21} e^{x\left(a_{11} / D_{1}\right)^{1 / 2}}+a_{23} e^{x\left(a_{33} / D_{3}\right)^{1 / 2}}\right]>0, \\
\bar{D}_{3} \nabla^{2} u_{3}^{*}-\partial u_{3}^{*} / \partial t+a_{31} u_{1}^{*}+a_{32} u_{2}^{*}-a_{33} u_{3}^{*} \\
=\varepsilon\left[a_{31} e^{x\left(a_{11} / D_{1}\right)^{1 / 2}}+a_{32} e^{x\left(a_{22} / D_{2}\right)^{1 / 2}}\right]>0 .
\end{array}\right\}
$$

Assume that $u_{1}^{*}, u_{2}^{*}$ and $u_{3}^{*}$ all attain their maximum values in the interior of $\mathbf{R}$, respectively at the points $\left(\mathbf{x}_{1}, t_{1}\right),\left(\mathbf{x}_{2}, t_{2}\right)$ and $\left(\mathbf{x}_{3}, t_{3}\right)$. Therefore

$$
\left.\frac{\partial u_{i}^{*}}{\partial t}\right|_{\left(\mathbf{x}_{i}, t_{i}\right)}=0,\left.\quad \nabla^{2} u_{i}^{*}\right|_{\left(\mathbf{x}_{i}, t_{i}\right)} \leqslant 0, \quad(i=1,2,3) .
$$

It is observed that if $u_{i}^{*}$ has a maximum on $\Omega \times(t=T)$, then $\partial u_{i}^{*} / \partial t \geqslant 0$ there. This only serves to strengthen the following inequalities. The substitution of (2.6) into (2.5) yields

$$
\left.\begin{array}{c}
-a_{11} u_{1}^{*}\left(\mathbf{x}_{1}, t_{1}\right)+a_{12} u_{2}^{*}\left(\mathbf{x}_{1}, t_{1}\right)+a_{13} u_{3}^{*}\left(\mathbf{x}_{1}, t_{1}\right)>0 \\
a_{21} u_{1}^{*}\left(\mathbf{x}_{2}, t_{2}\right)-a_{22} u_{2}^{*}\left(\mathbf{x}_{2}, t_{2}\right)+a_{23} u_{3}^{*}\left(\mathbf{x}_{2}, t_{2}\right)>0 \\
a_{31} u_{1}^{*}\left(\mathbf{x}_{3}, t_{3}\right)+a_{32} u_{2}^{*}\left(\mathbf{x}_{3}, t_{3}\right)-a_{33} u_{3}^{*}\left(\mathbf{x}_{3}, t_{3}\right)>0
\end{array}\right\}
$$

The assumption that $u_{1}^{*}, u_{2}^{*}$ and $u_{3}^{*}$ attain their maximum values at $\left(\mathbf{x}_{1}, t_{1}\right)$, $\left(\mathrm{x}_{2}, t_{2}\right)$ and $\left(\mathrm{x}_{3}, t_{3}\right)$ respectively, implies

$$
u_{l}^{*}\left(\mathbf{x}_{i}, t_{l}\right) \geqslant u_{l}^{*}\left(\mathbf{x}_{J}, t_{j}\right), \quad(i, j=1,2,3) .
$$

Inequalities (2.8) can be used to strengthen inequalities (2.7) to obtain

$$
\left.\begin{array}{l}
a_{11} u_{1}^{*}\left(\mathbf{x}_{1}, t_{1}\right)<a_{12} u_{2}^{*}\left(\mathbf{x}_{2}, t_{2}\right)+a_{13} u_{3}^{*}\left(\mathbf{x}_{3}, t_{3}\right), \\
a_{22} u_{2}^{*}\left(\mathbf{x}_{2}, t_{2}\right)<a_{21} u_{1}^{*}\left(\mathbf{x}_{1}, t_{1}\right)+a_{23} u_{3}^{*}\left(\mathbf{x}_{3}, t_{3}\right), \\
a_{33} u_{3}^{*}\left(\mathbf{x}_{3}, t_{3}\right)<a_{31} u_{1}^{*}\left(\mathbf{x}_{1}, t_{1}\right)+a_{32} u_{2}^{*}\left(\mathbf{x}_{2}, t_{2}\right) .
\end{array}\right\}
$$

The elimination of $u_{1}^{*}\left(\mathbf{x}_{1}, t_{1}\right)$ from (2.9) yields

$$
\left.\begin{array}{l}
\left(a_{12} a_{21}-a_{11} a_{22}\right) u_{2}^{*}\left(\mathbf{x}_{2}, t_{2}\right)+\left(a_{13} a_{21}+a_{11} a_{23}\right) u_{3}^{*}\left(\mathbf{x}_{3}, t_{3}\right)>0 \\
\left(a_{12} a_{31}+a_{11} a_{32}\right) u_{2}^{*}\left(\mathbf{x}_{2}, t_{2}\right)+\left(a_{13} a_{31}-a_{11} a_{33}\right) u_{3}^{*}\left(\mathbf{x}_{3}, t_{3}\right)>0 .
\end{array}\right\}
$$

Conditions (iv) of the theorem ensure that (2.10) is of the form (2.2). Application of the lemma to (2.10) gives

$$
\left(a_{11} a_{22}-a_{12} a_{21}\right)\left(a_{11} a_{33}-a_{13} a_{31}\right)<\left(a_{11} a_{23}+a_{13} a_{21}\right)\left(a_{11} a_{32}+a_{12} a_{31}\right) .
$$


Inequality (2.11) constitutes a contradiction of condition (v) of the theorem. Therefore the assumption that $u_{1}^{*}, u_{2}^{*}$ and $u_{3}^{*}$ all attain their maximum values in the interior of $\mathbf{R}$ is invalid. Hence at least one of the following holds,

$$
u_{1}^{*} \leqslant \max _{\partial \mathrm{R}} u_{1}^{*}, \quad u_{2}^{*} \leqslant \max _{\partial \mathrm{R}} u_{2}^{*}, \quad u_{3}^{*} \leqslant \max _{\partial \mathrm{R}} u_{3}^{*},
$$

where $\partial \mathbf{R}$ denotes the boundary of $\mathbf{R}$. By returning to the original concentrations we see that at least one of the following holds:

$$
\left.\begin{array}{l}
u_{1} \leqslant u_{1}+\varepsilon e^{x\left(a_{11} / D_{1}\right)^{1 / 2}} \leqslant \max _{\partial \mathbf{R}} u_{1}+\varepsilon \max _{\partial \mathbf{R}} e^{x\left(a_{11} / D_{1}\right)^{1 / 2}}, \\
u_{2} \leqslant u_{2}+\varepsilon e^{x\left(a_{22} / D_{2}\right)^{1 / 2}} \leqslant \max _{\partial \mathbf{R}} u_{2}+\varepsilon \max _{\partial \mathbf{R}} e^{x\left(a_{22} / D_{2}\right)^{1 / 2}}, \\
u_{3} \leqslant u_{3}+\varepsilon e^{x\left(a_{33} / D_{3}\right)^{1 / 2}} \leqslant \max _{\partial \mathbf{R}} u_{3}+\varepsilon \max _{\partial \mathbf{R}} e^{x\left(a_{33} / D_{3}\right)^{1 / 2}}
\end{array}\right\}
$$

Letting $\varepsilon$ approach zero yields

$$
u_{1} \leqslant \max _{\partial \mathbf{R}} u_{1} \text { or } u_{2} \leqslant \max _{\partial \mathbf{R}} u_{2} \text { or } u_{3} \leqslant \max _{\partial \mathbf{R}} u_{3},
$$

concluding the proof of the theorem.

Theorem 2.1 also holds if conditions (iv) and (v) are replaced by either of the following two sets of conditions:

$$
\left.\begin{array}{rr}
\text { (iv) } & a_{11} a_{22}>a_{12} a_{21}, \quad a_{22} a_{33}>a_{23} a_{32}, \\
\text { (v) } & \left(a_{11} a_{22}-a_{12} a_{21}\right)\left(a_{22} a_{33}-a_{23} a_{32}\right) \\
& \geqslant\left(a_{22} a_{13}+a_{23} a_{12}\right)\left(a_{22} a_{31}+a_{21} a_{32}\right)
\end{array}\right\}
$$

or

$$
\left.\begin{array}{ccc}
\text { (iv) })_{2} & a_{11} a_{33}>a_{13} a_{31}, & a_{22} a_{33}>a_{23} a_{32}, \\
\text { (v) } & \left(a_{11} a_{33}-a_{13} a_{31}\right)\left(a_{22} a_{33}-a_{23} a_{32}\right) \\
& \geqslant\left(a_{33} a_{12}+a_{32} a_{13}\right)\left(a_{33} a_{21}+a_{31} a_{23}\right) .
\end{array}\right\}
$$

As a direct consequence of substituting (1.4) into the above theorem, the corresponding maximum principle is obtained for (1.1). It has already been noted that conditions (iv) and (v) of Theorem 2.1 are trivially satisfied upon this substitution. Therefore the corresponding maximum principle for diffusion in the presence of three diffusion paths is as follows.

Definition. We say that $\left\{u_{1}, u_{2}, u_{3}\right\} \in H^{1}$ in $S$ if and only if

(i) $\left\{u_{1}, u_{2}, u_{3}\right\} \in C^{2}$ in $S$, and

(ii) $\left\{u_{1}, u_{2}, u_{3}\right\}$ satisfy (1.1) in $S$,

where $S$ is an arbitrary region of the $\mathrm{x}-t$ space, and $C^{2}$ denotes the set of functions which are continuous together with their derivatives up to second order. 
THEOREM 2.2. At least one of $u_{1}, u_{2}$ and $u_{3}$ attains its maximum on the boundary of $\mathbf{R}$ if the following hold:

(i) $\left\{u_{1}(\mathbf{x}, t), u_{2}(\mathbf{x}, t), u_{3}(\mathbf{x}, t)\right\} \in H^{1}$ if $\mathbf{x} \in \Omega, 0<t<T$,

(ii) $\left\{u_{1}(\mathbf{x}, t), u_{2}(\mathbf{x}, t), u_{3}(\mathbf{x}, t)\right\}$ are continuous if $(\mathbf{x}, t) \in \mathbf{R}$,

(iii) $\left\{u_{1}(\mathbf{x}, t), u_{2}(\mathbf{x}, t), u_{3}(\mathbf{x}, t)\right\} \geqslant 0$ if $(\mathbf{x}, t) \in \mathbf{R}$.

Theorem 2.1 can be extended to systems in which there are more than three diffusion paths. The detaiis are involved and become more difficult for larger systems. For $n$ diffusion equations, corresponding to the system (1.2), inequalities (2.9) may be generalized to

$$
a_{u} u_{i}^{*}\left(\mathbf{x}_{t}, t_{i}\right)<\sum_{\substack{j=1 \\ j \neq l}}^{n} a_{\imath} u_{j}^{*}\left(\mathbf{x}_{j}, t_{j}\right),
$$

for $i=1,2, \ldots, n$. For four diffusion paths a theorem similar to Theorem 2.1 can be proved in which conditions (iv) remain the same, condition (v) becomes a strict inequality, and if two further conditions are stipulated:

$$
\left.\begin{array}{l}
\left(a_{11} a_{44}-a_{14} a_{41}\right)\left(a_{11} a_{33}-a_{13} a_{31}\right)>\left(a_{41} a_{13}+a_{43} a_{11}\right)\left(a_{31} a_{14}+a_{34} a_{11}\right), \\
{\left[\left(a_{11} a_{44}-a_{14} a_{41}\right)\left(a_{11} a_{33}-a_{13} a_{31}\right)-\left(a_{41} a_{13}+a_{43} a_{11}\right)\left(a_{31} a_{14}+a_{34} a_{11}\right)\right]} \\
\times\left[\left(a_{11} a_{22}-a_{12} a_{21}\right)\left(a_{11} a_{33}-a_{13} a_{31}\right)-\left(a_{13} a_{21}+a_{23} a_{11}\right)\left(a_{12} a_{31}+a_{32} a_{11}\right)\right] \\
\geqslant\left[\left(a_{41} a_{12}+a_{42} a_{11}\right)\left(a_{11} a_{33}-a_{13} a_{31}\right)+\left(a_{41} a_{13}+a_{43} a_{11}\right)\left(a_{12} a_{31}+a_{32} a_{11}\right)\right] \\
\times\left[\left(a_{13} a_{21}+a_{23} a_{11}\right)\left(a_{31} a_{14}+a_{34} a_{11}\right)+\left(a_{11} a_{33}-a_{13} a_{31}\right)\left(a_{14} a_{21}+a_{24} a_{11}\right)\right] .
\end{array}\right\}
$$

There exist similar sets of conditions under which a maximum principle holds for systems with four diffusion paths. For further results concerning maximum principles for coupled parabolic systems the reader is referred to Dow [5], Dow [6], Dow and Vỷborný [7] and McNabb [11].

\section{Acknowledgement}

The authors wish to thank Alex McNabb (D.S.I.R, New Zealand) for suggesting this study and providing useful comments.

\section{References}

[1] E. C. Aifantis, "A new interpretation of diffusion in high diffusivity paths-a continuum approach", Acta Metall. 27 (1979), 683-691.

[2] E. C. Aifantis, "Continuum basis for diffusion in regions with multiple diffusivity", J. Appl. Phys. 50 (1979), 1334-1338. 
[3] E. C. Aifantis and J. M. Hill, "On the theory of diffusion in media with double diffusivity I. Basic mathematical results", Quart.J. Mech. Appl. Math. 33 (1980), 1-21.

[4] G. I. Barenblatt, Iu. P. Zheltov and I. N. Kochina, "Basic concepts in the theory of seepage of homogeneous liquids in fissured rocks", Appl. Math. Mechs. 24 (1960), 1286-1303.

[5] M. A. Dow, "Three-curves theorems for quasilinear inequalities", Duke Math. J. 41 (1974), 473-481.

[6] M. A. Dow, "Strong maximal principles for weakly coupled systems of quasilinear parabolic inequalities", J. Austral. Math. Soc. Ser. A 19 (1975), 103-120.

[7] M. A. Dow and R. Výbroný, "Maximum principles for some quasilinear second order partial differential equations", Rend. Sem. Mat. Univ. Padova 47 (1972), 331-351.

[8] J. M. Hill, "A discrete random walk model for diffusion in media with double diffusivity", $J$. Austral. Math. Soc. Ser. B 22 (1980), 58-74.

[9] J. M. Hill, "On the solution of reaction-diffusion equations", J. Inst. Math. Appl. 27 (1981), $177-194$.

[10] J. M. Hill and E. C. Aifantis, "On the theory of diffusion in media with double diffusivity II. Boundary value problems", Quart. J. Mech. Appl. Math. 33 (1980), 23-41.

[11] A. McNabb, "Comparison and existence theorems for multicomponent diffusion systems", $J$. Math. Anal. Appl. 3 (1961), 133-144.

[12] F. J. Molz, "Water transport through plant tissue: the apoplasm and symplasm pathways", $J$. Theoret. Biol. 59 (1976), 277-292.

[13] L. N Rubinstein, "Process of conduction of heat in heterogeneous media", Izv. Akad. Nauk SSR. Geogr. 12 (1948), 27-45. 